\title{
A Site-specific, Sustained-release Drug Delivery System for Aneurysmal Subarachnoid Hemorrhage
}

\author{
Daniel Hänggi $^{1}$ • Nima Etminan ${ }^{1}$ • Hans Jakob Steiger ${ }^{2}$ • Mark Johnson ${ }^{3}$. \\ M. Melissa Peet ${ }^{3}$. Tom Tice ${ }^{4} \cdot$ Kevin Burton ${ }^{4}$ - Bruce Hudson ${ }^{4} \cdot$ Michele Turner $^{4}$. \\ Angela Stella ${ }^{5}$ - Parissa Heshmati ${ }^{6}$ - Cara Davis ${ }^{4}$ - Herbert J. Faleck ${ }^{7}$. \\ R. Loch Macdonald ${ }^{7,8}$
}

Published online: 2 March 2016

(C) The American Society for Experimental NeuroTherapeutics, Inc. 2016

\begin{abstract}
Nimodipine is the only drug approved for use by the Food and Drug Administration for improving outcome after aneurysmal subarachnoid hemorrhage (SAH). It has less than optimal efficacy, causes dose-limiting hypotension in a substantial proportion of patients, and is administered enterally 6 times daily. We describe development of site-specific, sustainedrelease nimodipine microparticles that can be delivered once directly into the subarachnoid space or cerebral ventricles for potential improvement in outcome of patients with aneurysmal SAH. Eight injectable microparticle formulations of nimodipine in poly(DL-lactide-co-glycolide) (PLGA) polymers of varying composition were tested in vitro, and 1 was advanced into preclinical studies and clinical application. Intracisternal or intraventricular injection of nimodipine-PLGA microparticles in
\end{abstract}

Daniel Hänggi

daniel.haenggi@medma.uni-heidelberg.de

1 Department of Neurosurgery, University Medical Center Mannheim, Ruprecht-Karls-University Heidelberg, Germany,

Mannheim, Germany

2 Department of Neurosurgery, Medical Faculty, Heinrich-Heine-University, Düsseldorf, Germany

3 MPI Research, Mattawan, MI, USA

4 Evonik Industries, Birmingham, AL, USA

5 Pharmaceutical Associates, LLC, Tampa, FL, USA

6 Google, Mountain View, CA, USA

7 Edge Therapeutics, Inc., Berkeley Heights, NJ, USA

8 Division of Neurosurgery, St. Michael's Hospital, Labatt Family Centre of Excellence in Brain Injury and Trauma Research, Keenan Centre for Biomedical Science, Li Ka Shing Knowledge Institute, St. Michael's Hospital, Department of Surgery, University of Toronto, Toronto, ON, Canada rats and beagles demonstrated dose-dependent, sustained concentrations of nimodipine in plasma and cerebrospinal fluid for up to 29 days with minimal toxicity in the brain or systemic tissues at doses $<2 \mathrm{mg}$ in rats and $51 \mathrm{mg}$ in beagles, which would be equivalent of up to $612-1200 \mathrm{mg}$ in humans, based on scaling relative to cerebrospinal fluid volumes. Efficacy was tested in the double-hemorrhage dog model of SAH. Nimodipine-PLGA microparticles significantly attenuated angiographic vasospasm. This therapeutic approach shows promise for improving outcome after SAH and may have broader applicability for similar diseases that are confined to body cavities or spaces, are self-limited, and lack effective treatments.

Key Words Angiographic vasospasm · nimodipine · sustained release $\cdot$ subarachnoid hemorrhage . delayed cerebral ischemia.

\section{Introduction}

Many drugs have been studied but have failed to improve outcome after serious brain diseases such as ischemic stroke, traumatic brain injury, subarachnoid hemorrhage (SAH), and malignant brain tumors [1]. For some drugs, adverse effects may limit the dose that can be administered systemically to achieve therapeutic concentrations in the brain or cerebrospinal fluid (CSF). Strategies that have been used to circumvent this and that have had limited success include blood-brain barrier opening and use of transporters [2,3]. Another is to deliver drugs directly into the brain or subarachnoid space by injection, surgical implantation, or endonasal route [2]. Only one such formulation has been developed to date [4]. Limitations of subarachnoid drug delivery are that injection into the CSF may not produce adequate drug concentrations in the brain. Another is that most diseases require sustained drug concentrations for 
some time and there is limited ability to access the brain directly without invasive procedures that carry some risk.

An ideal disease to develop intracranial drug delivery for would be a serious, self-limited, subarachnoid-based disease. One example is aneurysmal $\mathrm{SAH}$, outcome from which remains poor with $35 \%$ of patients dying and many having permanent neurological and neurocognitive morbidity $[5,6]$. Furthermore, an important cause of morbidity after SAH is delayed cerebral ischemia (DCI), which does not occur until days after SAH and thus may permit some time to elapse before drug delivery [7]. Despite testing of $>50$ drugs, no pharmacologic treatment for improving outcome after $\mathrm{SAH}$ has been developed since nimodipine was approved for use in the USA in 1988 [8, 9].

Nimodipine, a dihydropyridine L-type calcium channel antagonist, has slight selective vasodilatory action on cerebral compared with systemic arteries, and is the only widely used drug for SAH [10]. Clinically, however, the dose that can be administered is limited because L-type calcium channels are located on arteries throughout the brain and body, and doses that dilate the cerebral arteries have some dilatory effect on systemic arteries, causing potentially deleterious adverse effects such as hypotension. Surgically placing pellets containing nicardipine, another dihydropyridine, into the subarachnoid space after aneurysmal SAH reduced DCI and cerebral infarction and improved outcome in experimental and clinical studies of aneurysmal SAH $[11,12]$. However, the pellets can only be implanted during a craniotomy conducted for aneurysm repair and currently at least $50 \%$ of aneurysms are repaired endovascularly. They are prepared with dichloromethane, a neurotoxin that may not be optimal for human use [11]. Given that nimodipine is effective at improving outcome, we sought to develop a formulation that could be administered either intracisternally after neurosurgical clipping of ruptured aneurysms, or intraventricularly to patients with a ventricular catheter who undergo endovascular aneurysm repair, or even neurosurgical clipping [13]. A sustained-release formulation was developed because intracranial access may be limited to the early phase after SAH, while nimodipine must be administered for 14-21 days [14]. We report on our initial experiments in rodents, followed by characterization of a unique, programmable, sustained release, injectable formulation of nimodipine in a dog model of SAH, and toxicity studies in rats and beagles.

\section{Methods}

Detailed methods and results are available online (Supplementary methods and results).

\section{Formulation Development and Release In Vitro}

Nimodipine has an asymmetric carbon at position 4 of one of the pyridine rings. The synthesis results in a racemic mixture of the 2 enantiomers that, in solid form, has 2 polymorphs and an amorphous form. Form 1 was a yellow crystal with a melting point of $124^{\circ} \mathrm{C}$. Form 2 was an almost white crystal with a melting point of $116^{\circ} \mathrm{C}$ and was a conglomerate when solid. The polymorphs were characterized by pycnometry, solubility measurements, differential scanning calorimetry, x-ray powder diffractometry, x-ray structure analysis, and infrared, Raman, and ${ }^{1} \mathrm{H}$-nuclear magnetic resonance spectroscopy (Supplementary methods and results). Microparticles consisting mainly of form 1 were used.

Microparticles were prepared by an oil/water emulsion microencapsulation technique using solvent extraction to precipitate the polymer, trap the drug, and harden the microparticles. This dispersion phase was combined with the continuous phase just prior to passing through the ceramic bead-filled column creating an emulsion that was then passed into an extraction bath containing deionized water. The extracted microparticles were collected on sieves, lyophilized, and stored at $-20{ }^{\circ} \mathrm{C}$. The dried microparticles were sterilized using gamma irradiation (25-30 kilogray) from a cobalt 60 source (Sterix Isomedix, Libertyville, IL, USA).

Release characteristics for 14 days in vitro were assessed for different formulations. Ten $\mathrm{mg}$ microparticles were weighed into a $50-\mathrm{ml}$ polypropylene tube and $20 \mathrm{ml}$ of sodium dodecyl sulfate/phosphate buffered saline (PBS) buffer was added. Tubes were incubated in a shaker water bath at $37^{\circ} \mathrm{C}$. Buffer was sampled at $1,3,6$, and $24 \mathrm{~h}$, and then daily for 14 days.

Nimodipine concentrations in sodium dodecyl sulfate/PBS buffer were measured by reverse-phase high-performance liquid chromatography and in plasma and CSF by liquid chromatography tandem mass spectroscopy using a United States Pharmacopea method (Agilent Technologies, Santa Clara, CA, USA, and Perkin Elmer, Waltham, MA, USA). Nimodipine binding to plastic from CSF samples was prevented by adding octyl-D-glucopyranoside at approximately $1.5 \%$ (weight/volume).

\section{Nimodipine Release In Vivo}

The low-viscosity hyaluronic acid used as a vehicle to prepare the nimodipine-poly(DL-lactide-co-glycolide) (PLGA) microparticles suspension was obtained from Fidia (Hyalgan; Abano Terme, Italy). In 1 arm of the in vivo experiments, nimodipine-PLGA microparticles injected into the cisterna magna were mixed with high-viscosity hyaluronic acid (Orthovisc, Bedford, MA, USA).

Formulations and pure nimodipine were implanted subcutaneously in 44 male Wistar rats instrumented with jugular vein cannulas, approximately 9 weeks of age. Neat nimodipine was prepared in $1.87 \%$ hydroxypropyl methylcellulose in PBS. The nimodipine reference solution and nimodipine-PLGA microparticle formulations 00447-098, 
00447-102, 00447-104, 00447-108, 00447-110, and 00447116 were administered via a single subcutaneous injection in the left hindlimb of each animal at doses of 20 (in $0.15 \mathrm{ml}$ ) or $200(0.7 \mathrm{ml}) \mathrm{mg} / \mathrm{kg}$. Blood samples were collected from2 alternating cohorts of 2 or 3 animals/cohort in the microparticle formulation-injected groups. Collection was immediately before injection and then 1,12 , and $24 \mathrm{~h}$ postdose, and on days $4,8,11$, and 15. After the last blood sample collection interval, the surviving animals were euthanized via carbon dioxide inhalation. Animals were euthanized by cervical dislocation and the carcasses were discarded.

\section{Toxicity Studies}

We next tested whether nimodipine-PLGA microparticles were toxic in rats and beagles. The primary objectives were to assess toxicity, determine a no observed adverse effect level (NOAEL), and measure pharmacokinetics. Rats [Crl:CD(SD), , 130-290 g, 190 rats, CD; Charles River, Portage, MI, USA) were randomly allocated with equal numbers of each sex ( $n=7-13$ per sex per group) to undergo intraventricular injection of equal volumes of $0.9 \% \mathrm{NaCl}$, placebo microparticles, or nimodipine microparticles at doses $0.33,1$, or $2 \mathrm{mg}$ nimodipine on day 1 and euthanasia on day 15 or 29 (Supplementary Table 1). These doses were selected based on preliminary maximum tolerated dose (MTD) studies showing that the MTD in rats was nimodipine-PLGA, $2 \mathrm{mg}$. These doses correspond to human doses of 200, 600, and $1200 \mathrm{mg}$, scaled on relative CSF volumes, assuming a $300-\mathrm{g}$ rat has a CSF volume of $150 \mu \mathrm{l}$ and a $70-\mathrm{kg}$ human has a CSF volume of $150 \mathrm{ml}$, with the maximum dose being the MTD [15]. Similar results are obtained scaling for body surface area [16]. Furthermore, we estimated a human dose of intracranial nimodipine-PLGA might be in the vicinity of $760 \mathrm{mg}$ because it is one-tenth of the human enteral dose over 21 days, given the approximately $10 \%$ bioavailability of enteral nimodipine $[17,18]$. Nimodipine and placebo microparticles were suspended in low-viscosity hyaluronic acid buffer prior to intraventricular injection. Animals were observed twice daily and examined on days 1 to 7, 14, 21, and 28. Functional observational battery, body weight, temperature, ophthalmoscopic examination, blood hematology, coagulation, clinical chemistry, urine samples, and plasma and CSF for nimodipine concentrations were assessed at various times (Supplementary methods and results).

Seventy-eight beagles ( $8-12 \mathrm{~kg}, n=3$ per sex per group; Marshall BioResources, North Rose, NY, USA) were randomly allocated to receive intraventricular or intracisternal injection of $0.9 \% \mathrm{NaCl}$, placebo microparticles, or nimodipine microparticles (Supplementary Table 2). For intraventricular injection, nimodipine or placebo microparticles were suspended in low-viscosity hyaluronic acid (Hyalgan) and for intracisternal injection they were suspended in high- viscosity hyaluronic acid (Orthovisc). The injection volume was $1.15 \mathrm{ml}$ and the dose of nimodipine was 17,51 , or $103 \mathrm{mg}$. These doses were selected based on preliminary MTD studies showing that the MTD in beagles was nimodipine-PLGA, $103 \mathrm{mg}$. This corresponds to human doses of 200,600 , and $1200 \mathrm{mg}$, scaled on relative CSF volumes, assuming a CSF volume of $12.5 \mathrm{ml}$ in a $12.5-\mathrm{kg}$ beagle and a CSF volume of $150 \mathrm{ml}$ in a $70-\mathrm{kg}$ human, with the maximum dose being the maximum feasible dose [15]. These doses yield a similar human equivalent dose based on body surface area or CSF volume scaling as the doses used in the rat toxicity study [16].

All animals were observed twice daily. Clinical and behavior examinations were conducted on days 1-7, 15, 21, and 29 [19]. Body weight was measured weekly and ophthalmoscopy was conducted before injections and prior to euthanasia. Indirect blood pressure, heart rate, electrocardiography, blood samples, and urine data were collected; also, plasma and CSF for nimodipine concentrations were collected at intervals (Supplementary methods and results).

\section{Dog Efficacy Study}

Forty mongrel dogs (16-23 kg, $n=4$ per sex per group; Marshall BioResources) were randomly allocated to undergo baseline cerebral angiography (day 1) and cisternal blood injection followed by treatment with placebo microparticles; placebo microparticles plus enteral nimodipine $(5.2 \mathrm{mg} / \mathrm{kg}$ daily for 21 days, equivalent to a human dose of $30 \mathrm{mg}$ every $4 \mathrm{~h}$ for a $60-\mathrm{kg}$ human, based on body surface area) [16]; nimodipine-PLGA microparticles, $40 \mathrm{mg}$ intracisternal; nimodipine-PLGA microparticles, $100 \mathrm{mg}$ intracisternal; or nimodipine-PLGA microparticles, $100 \mathrm{mg}$ intraventricular. These doses were selected in mongrel dogs because they were gave similar human equivalent doses to the higher doses tested in the beagle toxicity study. Dogs were euthanized on day 28 or 49. Dogs were anesthetized as described above, blood was collected, and blood pressure, temperature, heart rate, and oxygen saturation were monitored continuously while animals were under anesthesia. Blood gases were obtained before and after the injections. Cerebral angiography was performed through a 4-F catheter inserted into the femoral artery and advanced under fluoroscopic guidance into the proximal portion of one vertebral artery using a guidewire. A single arterial-phase anteroposterior angiogram was obtained by injection of up to $8 \mathrm{ml}$ contrast (diatrizoate meglumine $60 \%$; United States Pharmacopeia, Amersham Health, Princeton, NJ, USA). Images were captured digitally using identical exposure factors and magnification, and an internal magnification standard was included in every angiogram.

After angiography, dogs were turned prone, tilted $30^{\circ}$ head down and the cisterna magna was punctured percutaneously with a 14-gauge thin-walled spinal needle. CSF, $0.3 \mathrm{ml} / \mathrm{kg}$, 
was allowed to drain spontaneously, after which approximately $0.3-0.5 \mathrm{ml} / \mathrm{kg}$ of fresh, autologous, arterial, nonheparinized blood was withdrawn from the femoral catheter and injected into the cisterna magna at a rate of $5 \mathrm{ml} / \mathrm{min}$. Half of the blood was injected, followed by injection of nimodipine or placebo microparticles suspended in hyaluronic acid, followed by the remaining blood. The needle was withdrawn and the dogs were kept $30^{\circ}$ head down for $15 \mathrm{~min}$, after which the femoral catheter was removed, the artery ligated, and the incision closed.

For intraventricular injection, the blood was injected into the cisterna magna and then the dog was placed prone in a stereotactic unit. Nimodipine microparticles were injected into the right lateral ventricle using the same procedure as described in the beagle toxicity study.

On day 3, dogs were anesthetized as above and blood was injected into the cisterna magna using the same procedure as on day 1 except that microparticles were not injected. Angiography was repeated on days 8 and 15 . The diameter of the basilar artery was measured at 5 equally spaced points by 2 blinded observers and the means averaged. Angiographic vasospasm was assessed by comparing the diameters of basilar arteries from days 1,8 , and 15 , and the time course and severity of angiographic vasospasm was determined. In previous studies, as well as the current data, we found angiographic vasospasm was most severe in the basilar artery and was much less in the posterior communicating arteries and anterior circle of Willis [20]. Dogs were weighed weekly and examined daily for 14 days, including behavior examination (Supplementary methods and results) [19]. Indirect blood pressure was measured in awake dogs daily for the first 14 days and weekly thereafter.

Plasma and CSF were collected for determination of nimodipine concentration. Dogs were euthanized by an intravenous overdose of sodium pentobarbital. They were perfused through the heart with PBS followed by $10 \%$ neutral buffered formalin. The brain and spinal cord were excised and axial cross sections of the midbrain, pons, medulla, and cervical, thoracic, and lumbar spinal cord, and a coronal section of the cerebral hemispheres through the middle cerebral artery territory were taken, embedded in paraffin, and stained with hematoxylin and eosin. A section from each level was also stained with fluoro-jade B, activated caspase-3, or antithrombin III (Supplementary methods and results).

\section{Data Analysis and Statistics}

All data are mean $\pm \mathrm{SD}$. For the toxicity studies, we compared saline with placebo within each route of administration and time (15 or 29 day sacrifice), and placebo with nimodipinePLGA microparticles groups within each route of administration and time (Supplementary Tables 2 and 3). Leukocyte count and urinalysis results were transformed by log and rank transformation, respectively, prior to analysis. Groups were compared by ANOVA. If there was significant variance $(p<0.05)$, pair-wise comparisons were made after adjustment for multiple comparisons by the Holm-Sidak or Tukey methods [21]. The functional observation battery was analyzed by the Cochran-Mantel-Haenszel test. All statistical analysis was done in Stata (version 9; Stata Corp., College Station, TX, USA).

\section{Results}

\section{Formulation Development}

Eight nimodipine-PLGA microparticle formulations were prepared considering a specific product profile target (Supplementary methods and results). Their chemical/ physical stability, sterilizability, and release characteristics in vitro were determined. PLGA was chosen as the microparticle excipient because it is a biocompatible, bioabsorbable polymer with a long safety record in humans, even when used intracranially, and it is one of few parenteral polymers that can release drug over weeks [22].

Parameters that were varied in the formulations were lactide to glycolide ratio, polymer end group chemistry, molecular weight, and co-solvents. The resulting microparticles varied from $25 \%$ to $50 \%$ glycolide, with 50-70\% theoretical drug loading and mean particle sizes of approximately 70 $100 \mu \mathrm{m}$, all formulated with ethyl acetate solvent and $>99 \%$ purity and $>91 \%$ encapsulation efficiency (Table 1 ).

Three selected formulations did not change in drug content, main peak purity, and mean particle size after sterilization with $>25$ kGray gamma radiation (Supplementary Table 3). Sterilization did not change the microparticle release characteristics in vitro (Fig. 1; Supplementary Fig. 1). This result showed the ability to prepare formulations that released nimodipine with different initial bursts and cumulative release over 14 days. Formulations containing form 1 were the most stable and there was little-to-no impact when small amounts of form 2 were present (Fig. 2). Microparticles did not change significantly after storage for 1 month at $25{ }^{\circ} \mathrm{C}$, based on scanning electron microscopy and release characteristics in vitro (Table 2; Fig. 2).

\section{Release In Vivo}

Drug release from sustained-release formulations typically differs in vitro and in vivo. To characterize release profiles for nimodipine-PLGA formulations, 6 nimodipine-PLGA formulations ( $n=6$ per group) or pure nimodipine $(n=4)$ were injected subcutaneously in Wistar rats at doses of 20 or $200 \mathrm{mg} / \mathrm{kg}$. There was sustained release of nimodipine into plasma over time with all formulations, including with pure 
Table 1 Characteristics of 6 nimodipine-poly(DL-lactide-co-glycolide) formulations

\begin{tabular}{|c|c|c|c|c|c|c|c|c|}
\hline Number & $\begin{array}{l}\text { Polymer composition } \\
\text { (lactide:glycolide } \\
\text { ratio, end group) }\end{array}$ & $\begin{array}{l}\text { Theoretical } \\
\text { drug content } \\
\text { (weight \%) }\end{array}$ & $\begin{array}{l}\text { Actual drug } \\
\text { content } \\
\text { (weight \%) }\end{array}$ & $\begin{array}{l}\text { Encapsulation } \\
\text { efficiency (\%) }\end{array}$ & $\begin{array}{l}\text { Main peak } \\
\text { purity }(\%)\end{array}$ & $\begin{array}{l}\text { Particle } \\
\text { size }(\mu \mathrm{m})\end{array}$ & $\begin{array}{l}\text { Residual } \\
\text { solvent } \\
\text { (weight \%) }\end{array}$ & $\begin{array}{l}\text { Residual } \\
\text { water } \\
\text { (weight \%) }\end{array}$ \\
\hline 00447-098 & $65: 35$, ester & 65.0 & $62 \forall 1.3$ & 95.4 & 98.9 & 37.2 & 0.56 & 0.39 \\
\hline 00447-102 & $65: 35$, acid & 65.0 & $61 \forall 0.7$ & 94.5 & 99.9 & 36.9 & 0.67 & 0.31 \\
\hline 00447-104 & $75: 25$, acid & 65.0 & $56 \forall 0.4$ & 86.6 & 99.9 & 39.1 & 0.12 & 0.38 \\
\hline 00447-106 & $75: 25$, acid & 65.0 & $63 \forall 1.5$ & 96.8 & 98.8 & 38.1 & 0.64 & 0.37 \\
\hline 00447-108 & 50:50, acid & 65.0 & $64 \forall 0.3$ & 98 & 99.9 & 34.7 & 0.93 & 0.24 \\
\hline 00447-110 & $65: 35$, ester & 50.0 & $49 \forall 0.3$ & 97.6 & 99.9 & 38.8 & 0.37 & 0.49 \\
\hline 00447-116 & $65: 35$, ester & 66.5 & $61 \forall 1.2$ & 91.3 & 99.9 & 30.9 & 0.37 & 0.29 \\
\hline
\end{tabular}

Data are means $\pm \operatorname{SD}(n=3$ per measurement $)$

nimodipine, which was likely owing to its high lipid solubility and subsequent prolonged residence in subcutaneous fat (Fig. 3). There was a poor relationship between the time course of release in vitro and in vivo, although the order of release was similar. We reasoned that release in vitro might be more reflective of how the formulation would release in the aqueous subarachnoid space, rather than the fatty subcutaneous space. Based on this we selected for preclinical and clinical development a formulation that was stable and had predetermined release characteristics in vitro (00447-108). Injectability of microparticles was found to require at least an 18-gauge and, reliably, a 14-gauge needle and suspension in hyaluronic acid (Supplementary methods and results).

\section{Toxicity Studies}

All rats survived to sacrifice. There were no effects of nimodipine-PLGA microparticles on clinical observations,

\section{Cumulative Release In Vitro}

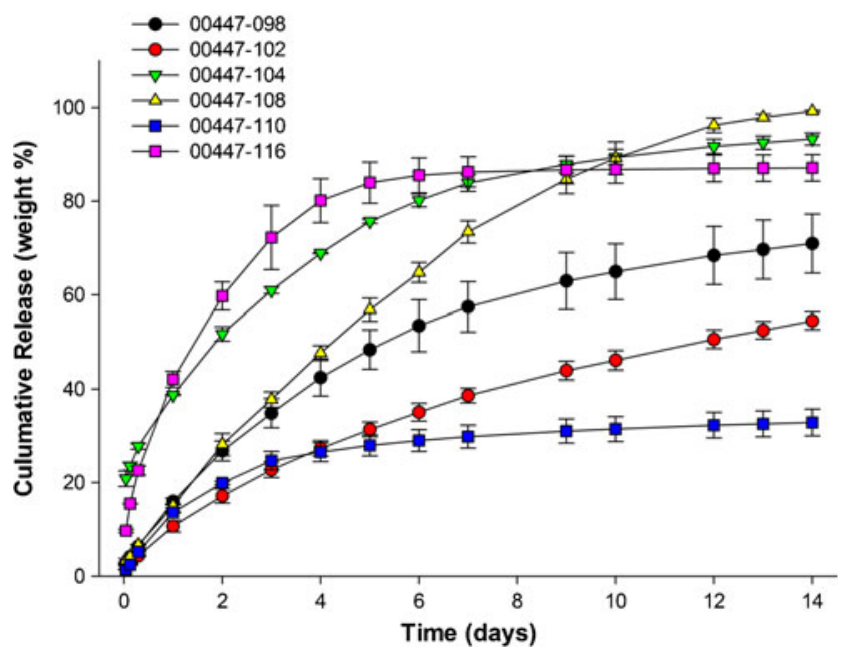

Fig. 1 Cumulative release of nimodipine in vitro from 6 nimodipinepoly(DL-lactide-co-glycolide) microparticle formulations under optimized sink conditions. Values are means $\pm \mathrm{SD}(n=3$ per measurement) neurobehavioral evaluations, body weight, food consumption, ophthalmoscopy, hematology, coagulation, clinical chemistry, urinalysis, organ weight, or macroscopic pathology. The only potential nimodipine-PLGA microparticle-related microscopic finding consisted of more frequent and severe intracranial hemorrhage at day 15 , usually at the injection site in the brain of rats injected with $2 \mathrm{mg}$ nimodipine-PLGA microparticles (Fig. 4). This finding was reversible as no differences were observed between groups by day 29 .

Histopathologic changes, including reactive gliosis, hydrocephalus, pigmented and foamy macrophages, focal mineralization and vacuolation, at or near the injection site at both times were observed in all groups (Fig. 4). The changes were thought to be related to the injection procedure, based on the lack of dose response and the similar incidence in all groups.

Mean plasma nimodipine concentrations at $48 \mathrm{~h}$ increased with increasing dose, and ranged from 0.88 to $10.6 \mathrm{ng} / \mathrm{ml}$ at $48 \mathrm{~h}$ (Fig. 5). Plasma concentrations after administration of $2 \mathrm{mg}$ nimodipine-PLGA microparticles were measurable on day 15 but not on day 29. Mean plasma maximum observed concentration $\left(\mathrm{C}_{\max }\right)$ and area under the curve from the time of dosing to the time of the last observation $\left(\mathrm{AUC}_{0-\mathrm{t}}\right)$ after injection of $2 \mathrm{mg}$ nimodipine-PLGA microparticles were, respectively, $40.5 \mathrm{ng} / \mathrm{ml}$ and $3902 \mathrm{ng}^{*} \mathrm{~h} / \mathrm{ml}, 5.7 \mathrm{ng} / \mathrm{ml}$ and $901 \mathrm{ng} * \mathrm{~h} /$ $\mathrm{ml}$ after injection of $1 \mathrm{mg}$, and $1.9 \mathrm{ng} / \mathrm{ml}$ and $177 \mathrm{ng} * \mathrm{hr} / \mathrm{ml}$ after injection of $0.33 \mathrm{mg}$ nimodipine-PLGA microparticles. Nimodipine was only measureable in 2 CSF samples obtained on day 3 , secondary to difficulty sampling CSF. The NOAEL was $2 \mathrm{mg}$.

In the beagle toxicity study, there were 2 mortalities and otherwise no clinical findings, changes in body weight, heart rate or blood pressure, clinical pathology, electrocardiography, or macroscopic observations (Supplementary Fig. 2). Two males were euthanized in extremis prior to the completion of the study. Both received $103 \mathrm{mg}$ nimodipine-PLGA microparticles intracisternally. They were euthanized on days 10 and 15 . The beagle euthanized on day 10 was found on microscopy to have the fourth ventricle of the brain expanded by granulomatous inflammation admixed with subacute/chronic 


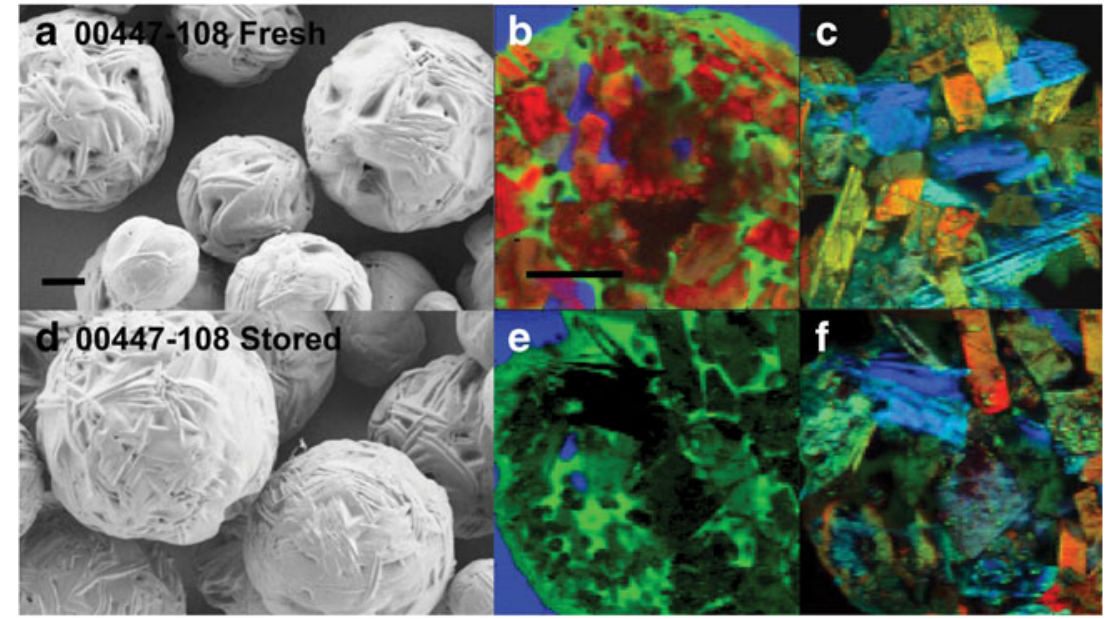

Fig. 2 (A, D, G, J) Scanning electron microscopy and (B, C, E, F, H, I, K, L) Raman spectroscopy of (A-C, G-I) fresh nimodipine microparticles or (D-F, J-L) the same formulations after storage at $30-35{ }^{\circ} \mathrm{C}$ for 30 days. Formulation 00447-108 showed no change in morphology or spectroscopy (top 2 rows, A-F), whereas 00447-110 showed agglomeration of particles after storage and change in nimodipine from amorphous to form 2 (scale bar $=20 \mu \mathrm{m})$. (B, E, H, and $\mathrm{K}$ ) Red is nimodipine, green is poly(DL-lactide-co-glycolide), and blue is epoxy in Raman spectroscopy. $(\mathrm{C}, \mathrm{F}, \mathrm{I}$, and $\mathrm{L}$ ) Red is nimodipine form 1, green is amorphous nimodipine and blue is nimodipine form 2 in Raman spectroscopy inflammation that infiltrated the lining of the fourth ventricle and choroid plexus. The inflammation surrounded moderate amounts of translucent, round, space-occupying foreign material. No foreign material or granulomatous inflammation was observed within the brain stem, midbrain, or cerebellum. Within the subjacent brain stem was an area of mild cavitation with rarefaction and edema of the neuropil, multifocal minimal hemorrhage, and minimal axonal/myelin degeneration with presence of spheroids, suggesting at some point an injection had penetrated the brainstem. In the beagle euthanized on day 15 , the fourth ventricle of the brain contained minimal translucent foreign material surrounded by minimal granulomatous/subacute/chronic inflammation. No foreign material or granulomatous inflammation was observed within the brain stem, midbrain, or cerebellum.
In general, microscopic findings were evident in the brain, spinal cord, and heart of some beagles (Fig. 6). Intraventricular or intracisternal injection caused a granulomatous foreign body type reaction in the cerebral ventricles and subarachnoid space. This inflammation and/or the injection procedure often lead to a disruption of the ependymal lining and subsequent extension of the inflammation and/or foreign material into the adjacent periventricular neuropil. The inflammatory reaction and presence of foreign material was observed after injection of any amount of PLGA microparticles, but the incidence and severity was related to dose. The periventricular extension of the inflammation was more prominent in animals receiving intraventricular injections. The foreign material and inflammatory reaction was incompletely resolved by day 29. Inflammation, fibrosis, cardiac myofiber

Table 2 Characteristics and differential scanning calorimetry of 4 nimodipine-poly(DL-lactide-co-glycolide) formulations after storage for 30 days

\begin{tabular}{lllllllll}
\hline Number & $\begin{array}{l}\text { Storage } \\
\text { condition }\left({ }^{\circ} \mathrm{C}\right)\end{array}$ & $\begin{array}{l}\text { Average drug } \\
\text { content }(\text { weight } \%)\end{array}$ & $\begin{array}{l}\text { Encapsulation } \\
\text { efficiency }(\%)\end{array}$ & $\begin{array}{l}\text { Particle } \\
\text { size }(\mu \mathrm{m})\end{array}$ & $\begin{array}{l}\text { Drug } \\
\text { purity }(\%)\end{array}$ & $\mathrm{T}_{\mathrm{g}}\left({ }^{\circ} \mathrm{C}\right)$ & $\begin{array}{l}\text { Main } \\
\text { melt }\left({ }^{\circ} \mathrm{C}\right)\end{array}$ & $\begin{array}{l}\text { Second } \\
\text { melt }\left({ }^{\circ} \mathrm{C}\right)\end{array}$ \\
\hline $00447-098$ & 4 & 61.2 & $94.2 \forall 4.7$ & 74.5 & 99.5 & 34.1 & 126.1 & No peak \\
$00447-098$ & 25 & 63.2 & $97.2 \forall 2.9$ & 77.8 & 99.6 & 42.1 & 126.5 & 113.8 (tiny peak) \\
$00447-098$ & $30-35$ & 66.2 & $101.8 \forall 2.1$ & 95.7 & 99.8 & 49 & 125.8 & 113.5 (tiny peak) \\
$00447-102$ & 4 & 62.8 & $96.6 \forall 3.3$ & 63.5 & 99.4 & 34.6 & 125 & 114.2 (tiny peak) \\
$00447-102$ & 25 & 57.6 & $88.6 \forall 8.3$ & 64.9 & 99.6 & 38 & 125 & 114.6 \\
$00447-102$ & $30-35$ & 65 & $100.1 \forall 0.5$ & 70.3 & 99.8 & 48.8 & 125 & 114.6 \\
$00447-108$ & 4 & 63 & $96.9 \forall 9.5$ & 79.3 & 99.7 & 35 & 126.5 & No peak \\
$00447-108$ & 25 & 62.8 & $96.6 \forall 10.4$ & 76.8 & 99.8 & 39.6 & 126.1 & No peak \\
$00447-108$ & $30-35$ & 68 & $104.6 \forall 1.6$ & 79.8 & 99.8 & 49.44 & 126.5 & No peak \\
$00447-110$ & 4 & 52.1 & $104.3 \forall 2.5$ & 59.2 & 98.1 & 19.6 & 122 & 112.3 \\
$00447-110$ & 25 & 45.5 & $91.0 \forall$ & 127.4 & 99.7 & 33.9 & 112.7 & 122.4 \\
\hline
\end{tabular}




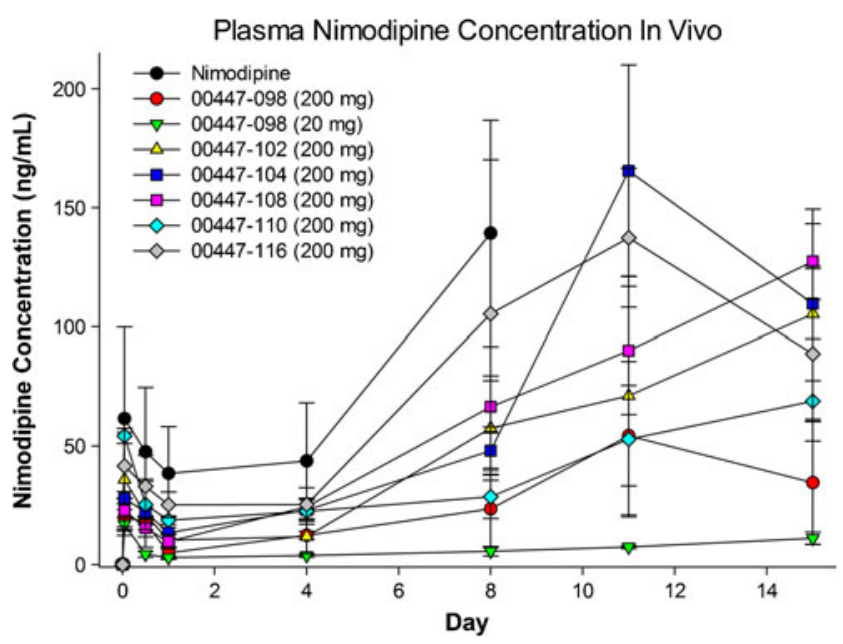

Fig. 3 Nimodipine plasma concentrations after subcutaneous injection of nimodipine $(n=4)$ or different nimodipine-poly(DL-lactide-coglycolide) formulations in rats. Values are means \pm SD $(n=2-3$ per measurement from groups of 6 rats)

degeneration/necrosis, and/or hypertrophy/hyperplasia of the tunica media of small-to-medium-sized arteries of the left ventricle and interventricular septum of the heart were observed primarily in animals receiving nimodipine microparticles by intracisternal injection and 1 high dose $(103 \mathrm{mg})$ male receiving intraventricular injection and euthanized on day 15 . No abnormal findings were noted in the intraventricular injected animals on day 29. Minimal left ventricular cardiac inflammation and fibrosis was seen in only 1 high-dose intracisternal injected male, suggesting partial resolution of the microscopic findings in the heart by day 29 .
Mean plasma nimodipine concentrations following intraventricular administration of nimodipine microparticles in beagles increased gradually during the first $48 \mathrm{~h}$ and then remained generally constant until 336 h (day 15; Fig. 7, Supplementary Table 4). Nimodipine was still detected at $672 \mathrm{~h}$ in all groups receiving nimodipine-PLGA microparticles (day 29). Plasma $\mathrm{C}_{\max }$ and $\mathrm{AUC}_{0-\mathrm{t}}$ increased with increasing dose. CSF nimodipine concentrations increased in a dosedependent fashion and were highest at the 48-h measurement (Fig. 7). Based upon the results of this study, a NOAEL of $51 \mathrm{mg}$ nimodipine-PLGA microparticles administered intraventricularly in the beagle was established.

\section{Mongrel Dog Efficacy Study}

One dog injected with nimodipine-PLGA microparticles intraventricularly $(100 \mathrm{mg})$ was euthanized on day 25 , and 1 in the placebo microparticle plus enteral nimodipine group was euthanized on day 35 . Both animals were well in the days leading up to CSF sampling after which they did not awaken. The cause of death was brainstem injury from aspiration of CSF from the cisterna magna.

Clinical examination showed similar findings in all groups with decreased appetite and activity within the first 5 days of SAH, followed by recovery (Supplementary Fig. 3) [19].

Mean blood pressures varied over time within groups and between groups at different times (Fig. 8). The lack of relationship between plasma nimodipine concentration and blood pressure suggests that intracranial administration of nimodipine-PLGA microparticles does not produce sufficient

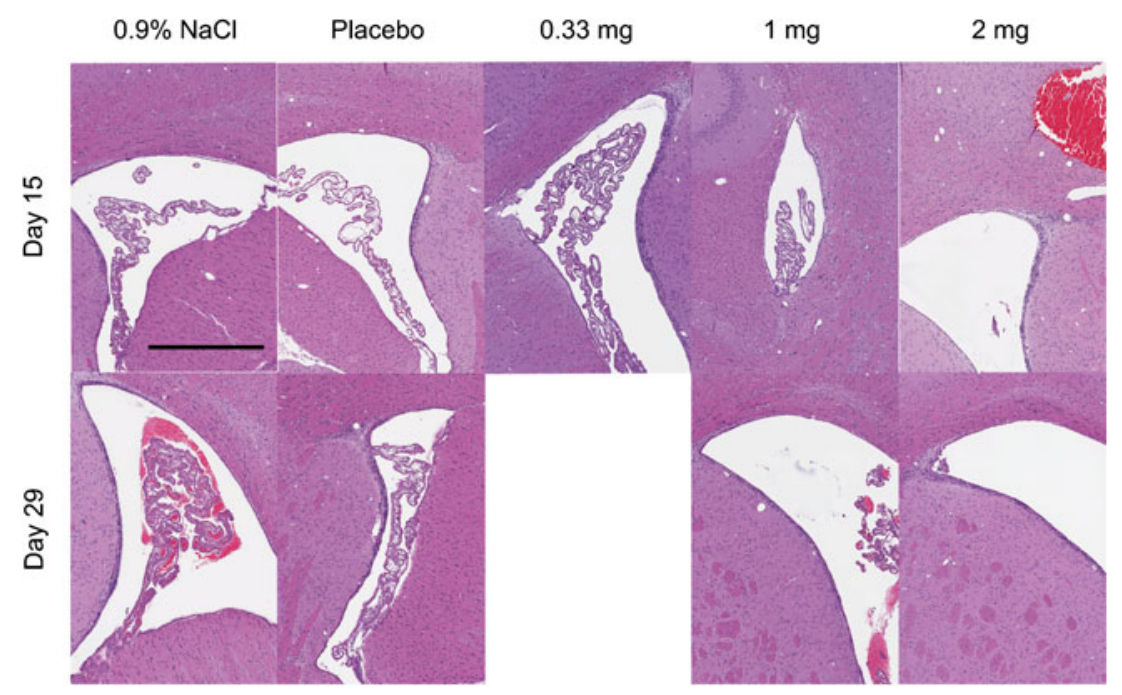

Fig. 4 Histologic (hematoxylin and eosin) effects of nimodipine microparticles in rats sacrificed 15 (top row) or 29 days (bottom row) after intraventricular injection of $0.9 \% \mathrm{NaCl}$, placebo microparticles or nimodipine-poly(DL-lactide-co-glycolide) (PLGA) microparticles $(0.33$, 1 , or $2 \mathrm{mg}$ nimodipine). There was reactive gliosis, hydrocephalus, pigmented and foamy macrophages, focal mineralization, and vacuolation after injection of microparticles that was not related to dose. More intracranial hemorrhage was observed 15 days after injection of nimodipine-PLGA microparticles, $2 \mathrm{mg}$, that partly resolved by 29 days. Scale bar $=500 \mu \mathrm{m}$ 


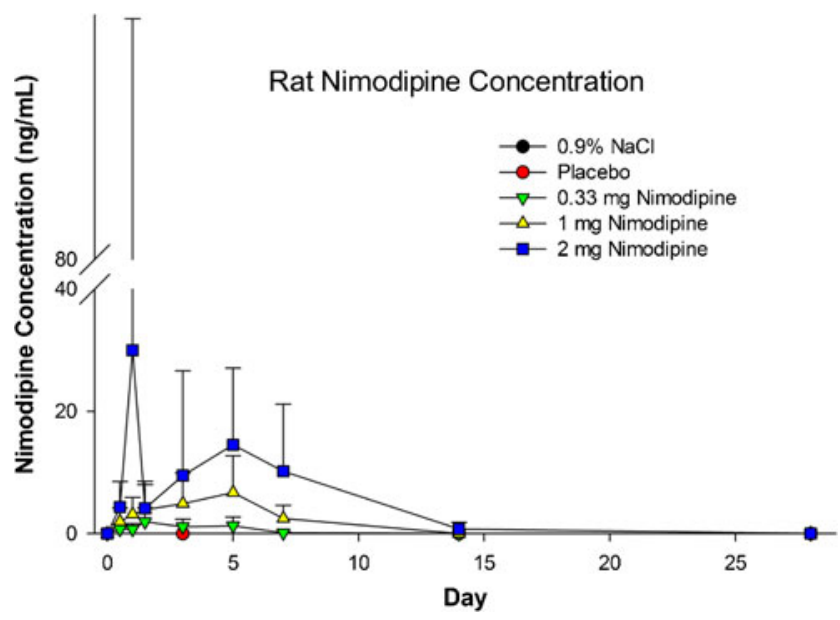

Fig. 5 Plasma concentration of nimodipine in rats after intraventricular injection of $0.9 \% \mathrm{NaCl}$, placebo microparticles or nimodipine-poly(DLlactide-co-glycolide) microparticles $(0.33,1$, or $2 \mathrm{mg}$ nimodipine). Values are means $\pm \operatorname{SD}(n=7-32$ per measurement $)$

systemic exposure or fluctuations in plasma concentrations to produce substantial changes in blood pressure.

Angiography showed significant reductions in basilar artery diameter 8 and 15 days after SAH in dogs treated with placebo microparticles with or without nimodipine (ANOVA, $p=0.001$, days 8 and 15 significantly different from day 1 ; $p<0.05$, Tukey test; Fig. 9, Supplementary Table 5). When comparing the percent reduction in basilar artery diameter between groups at day 8 , the group treated with $100 \mathrm{mg}$ nimodipine-PLGA microparticles intraventricularly had significantly less arterial narrowing than the placebo microparticle groups with or without enteral nimodipine $(p<0.009$,
ANOVA, Holm-Sidak). When comparing the percent reduction in basilar artery diameter between groups at day 15, the groups treated with $40 \mathrm{mg}$ nimodipine-PLGA microparticles intracisternal or $100 \mathrm{mg}$ intraventricularly had significantly less arterial narrowing than the placebo microparticle groups with or without enteral nimodipine $(p<0.009$, ANOVA, Holm-Sidak).

Plasma nimodipine concentrations increased in a dosedependent fashion to peak $36 \mathrm{~h}$ to 4 days after administration of nimodipine-PLGA microparticles intracisternally or intraventricularly (Fig. 8). Nimodipine was detected in plasma 21 days after administration of nimodipine-PLGA microparticles. The plasma concentration after administration of $100 \mathrm{mg}$ nimodipine-PLGA microparticles was qualitatively similar to that achieved with enteral nimodipine. Animals treated with enteral nimodipine had very low concentrations of nimodipine in CSF that were highest on day 3 (1.56 $\forall$ $1.17 \mathrm{ng} / \mathrm{ml}, n=7)$. Nimodipine concentrations were approximately 1000 -fold higher in animals treated with nimodipinePLGA microparticles.

Dogs were euthanized on day 28 or 49 ( $n=4$ per group per day). Microscopic changes in the brains consisted of granulomatous inflammation (aggregates of giant cells engulfing foreign material), subacute/chronic inflammation (mixed inflammatory infiltrate), fibroplasia, hemorrhage, and pigmented macrophages. These findings were mostly located in the meninges of pons, medulla, and, to a lesser extent, the cervical subarachnoid space. They were more prominent at the 28 day time and had a lower incidence and severity at day 49 .

Fluoro-jade B staining did not reveal any degenerative/ necrotic neurons. Apoptotic neurons were not detected in
Fig. 6 Histologic (hematoxylin and eosin) effects of nimodipine microparticles in beagles sacrificed 15 (top row) or 29 days (bottom row) after

intraventricular injection of $0.9 \%$ $\mathrm{NaCl}$, intraventricular nimodipine-poly(DL-lactide-coglycolide) (PLGA) microparticles (17, 51, or $103 \mathrm{mg}$ nimodipine), or intracisternal nimodipinePLGA microparticles (103 mg nimodipine). Granulomatous foreign body type reaction was noted in the cerebral ventricles and subarachnoid space after injection of any of the microparticle formulations although the incidence and severity was related to dose. The presence of the foreign material and inflammatory reaction was incompletely resolved by day 29. Scale bar $=500 \mu \mathrm{m}$

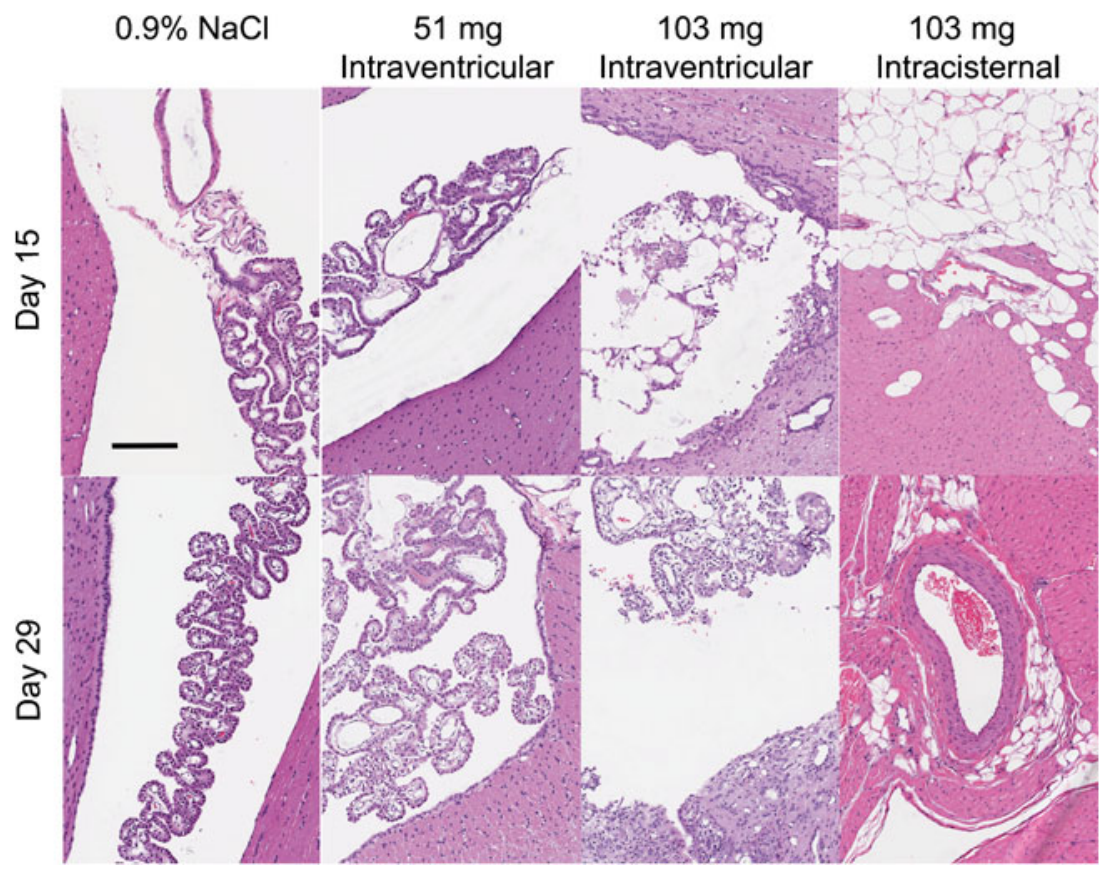




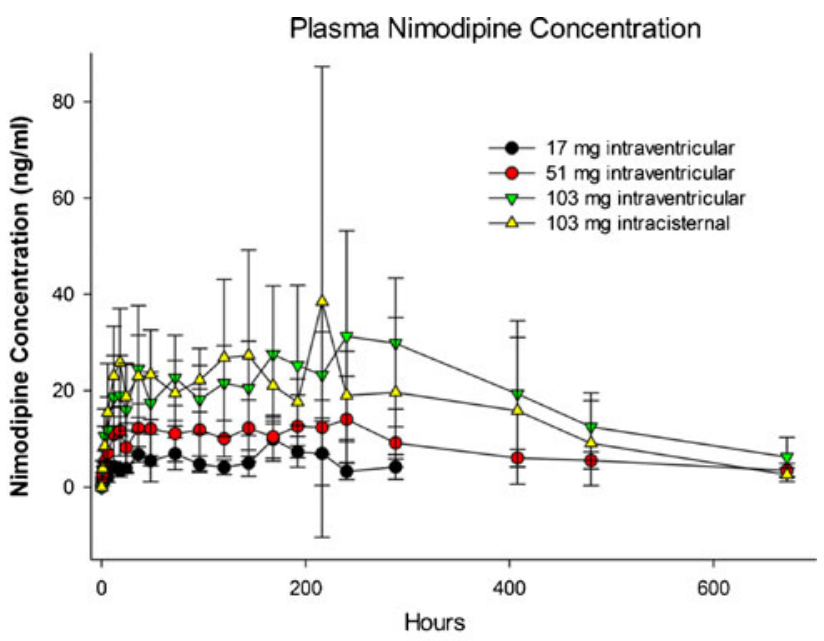

Cerebrospinal Fluid Nimodipine Concentration

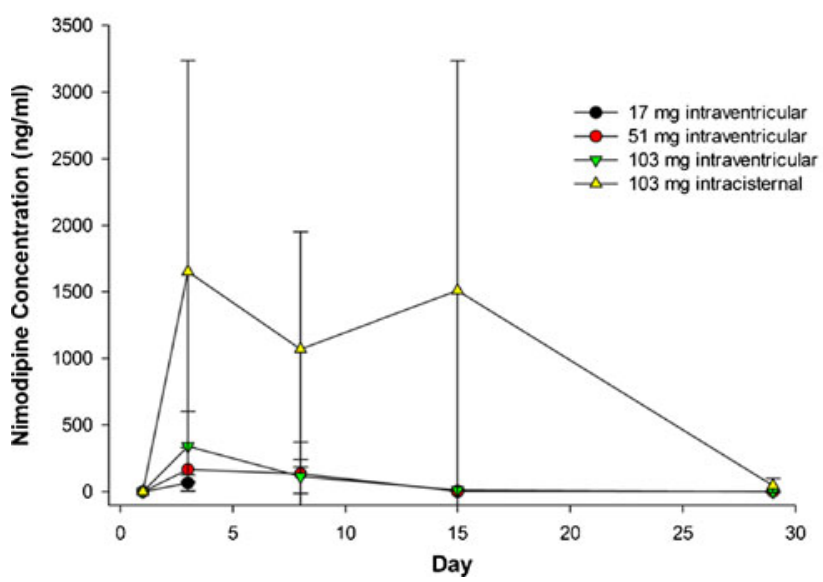

Fig. 7 Plasma (top) and cerebrospinal fluid (bottom) concentration of nimodipine in beagles after intraventricular or intracisternal injection of nimodipine-poly(DL-lactide-co-glycolide) microparticles $(17,51$, or $103 \mathrm{mg}$ nimodipine). Values are means $\pm \mathrm{SD}$

any animals by immunoreactivity to activated caspase- 3 . Apoptosis was detected in glial cells evenly distributed throughout the brain. Microthrombi were noted in 2 dogs in the placebo microparticle, 1 in the placebo microparticle plus enteral nimodipine, 1 in the $40 \mathrm{mg}$ intracisternally, 1 in the $100 \mathrm{mg}$ intracisternally, and 2 in the $100 \mathrm{mg}$ intraventricular nimodipine-PLGA microparticle group. Microthrombi were limited to the cerebrum with too few microthrombi observed to conduct statistical analysis.

\section{Discussion}

We report the novel development of a sustained release formulation that releases nimodipine in high concentrations into the CSF for up to 21 days after intracisternal or intraventricular delivery. The concentrations of nimodipine in plasma remain below those associated with hypotension and the formulation is injectable and manufactured by standards acceptable for use in humans. The efficacy, assessed by angiographic vasospasm, was greater than enteral nimodipine in dogs. In rats, there was more intracranial hemorrhage in those injected with nimodipine-PLGA microparticles, $2 \mathrm{mg}$, although this was reversible and had no observable effects. Importantly, this was not observed in beagles. Nimodipine has fibrinolytic activity that may contribute to its efficacy and could theoretically cause hemorrhage [7, 23]. However, there is no evidence that nimodipine is associated with hemorrhage in humans with $\mathrm{SAH}$, even after intracisternal or intraventricular administration [9]. Dose-dependent and reversible granulomatous inflammation was observed after injection of nimodipine-PLGA microparticles, a known reaction to PLGA that was without any sequelae in toxicity studies and in decades of use in humans.

Poor outcome after SAH is believed to result mainly from early brain injury and DCI. DCI is hypothesized to be due to a combination of angiographic vasospasm, microcirculatory dysfunction, cortical spreading ischemia, microthromboembolism, and delayed effects of early brain injury [7]. Nimodipine was noted to be a somewhat selective cerebral artery vasodilator that was studied in patients with SAH in order to reduce angiographic vasospasm. In the clinical trials, however, no or only a minimal decrease in angiographic vasospasm was observed in patients treated with nimodipine. Despite this and unlike other vasodilator drugs $[24,25]$, it improved clinical outcome. In addition to being a potent vasodilator, even of arteries with angiographic vasospasm $[12,26]$, it was later noted that nimodipine inhibited other delayed effects of SAH that may contribute to DCI, such as cortical spreading ischemia [27], and that it had fibrinolytic activity that could reduce microthromboemboli [23]. Other vasodilators may not have these pleiotrophic effects [28]. One hypothesis is that nimodipine improved outcome after SAH because it reduced these complications of SAH but that in the doses administered the effects were not measureable in clinical trials.

There have been prior reports of sustained-release formulations of dihydropyridines and other drugs administered into the subarachnoid space for SAH [12, 13, 29, 30]. While there has been some evidence that these may be effective, these formulations have had limitations, including lack of characterization of pharmacokinetics, stability, and injectability, use of materials with known or unknown toxicity, and limited data on efficacy of the active drug. The current work allowed translation of a sustainedrelease nimodipine formulation from preclinical to clinical use by using materials with known brain biocompatibility, methods that allow programming of the polymers for desired release characteristics, stability, and commercial scalability, and a known active pharmacologic agent. 


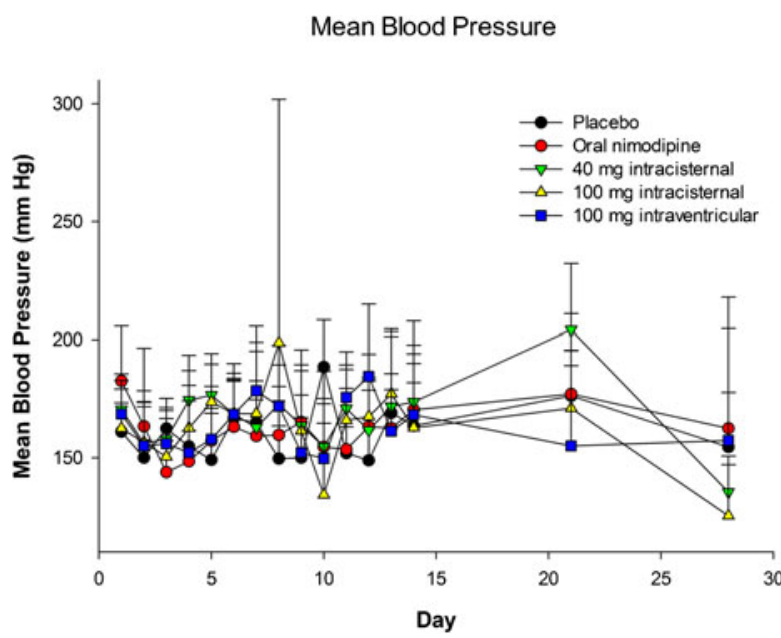

Fig. 8 Mean blood pressure (left), plasma (middle), and cerebrospinal fluid (right) nimodipine concentrations in mongrel dogs after subarachnoid hemorrhage treated with intracisternal placebo microparticles without $(n=8)$ or with enteral nimodipine ( $n=8,5.2 \mathrm{mg} / \mathrm{kg}$ daily for 21 days), intracisternal nimodipine-poly(DL-lactide-co-glycolide) (PLGA) microparticles $(n=8,40 \mathrm{mg})$, intracisternal nimodipine-PLGA microparticles $(n=6,100 \mathrm{mg})$, or intraventricular nimodipine-PLGA microparticles $(n=8,100 \mathrm{mg})$. Mean blood pressure varied significantly in dogs treated with $40 \mathrm{mg}$ intracisternal nimodipine-PLGA microparticles $(p<0.001$, ANOVA, pairwise differences between day 21 and each of days 2, 3, 7, 9, 10,12 , and 28, and between day 28 and each of days 4, 5, and 14, HolmSidak). There also was significant variance in mean blood pressure in the

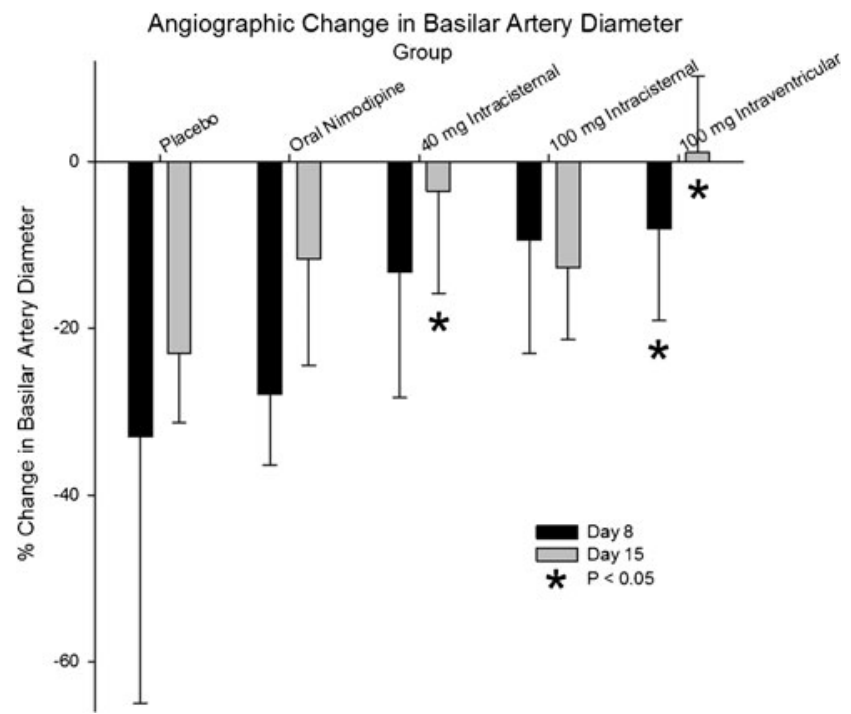

Fig. 9 Percent change in basilar artery diameter 8 and 15 days after subarachnoid hemorrhage in dogs treated with intracisternal placebo microparticles without $(n=7)$ or with enteral nimodipine $(n=8,5.2 \mathrm{mg} /$ $\mathrm{kg}$ daily for 21 days), intracisternal nimodipine- poly(DL-lactide-coglycolide) (PLGA) microparticles $(n=8,40 \mathrm{mg})$, intracisternal nimodipine-PLGA microparticles $(n=6,100 \mathrm{mg})$, or intraventricular nimodipine-PLGA microparticles $(n=8,100 \mathrm{mg})$. There was significantly less angiographic vasospasm at 8 days after treatment with $100 \mathrm{mg}$ nimodipine-PLGA microparticles intraventricularly, or at 15 days after treatment with $40 \mathrm{mg}$ intracisternal or $100 \mathrm{mg}$ intraventricular nimodipine-PLGA microparticles $(p<0.05$, ANOVA). Values are means \pm SD
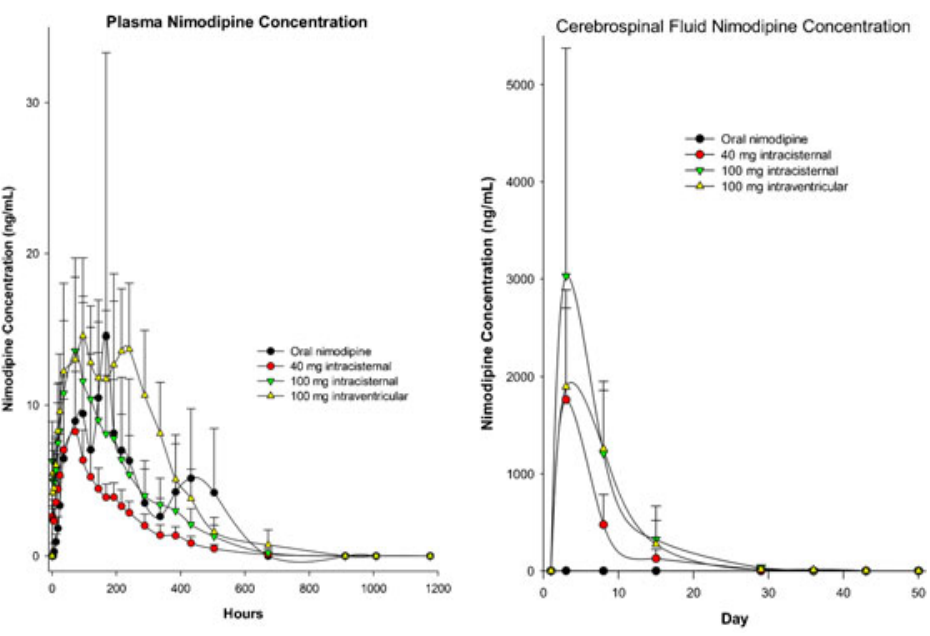

group treated with $100 \mathrm{mg}$ intracisternal nimodipine-PLGA microparticles ( $p<0.019$, no pairwise differences). When comparing between groups over time, there was significant variance in blood pressure on day 5 but no pairwise differences between groups (ANOVA, $p=0.033$ ), on day 10 , with pairwise differences between placebo and $100 \mathrm{mg}$ intracisternal or intraventricular (ANOVA, $p=0.004$; Holm-Sidak, $p<0.05$ ), and on day 21 , with pairwise difference between $40 \mathrm{mg}$ intracisternal and $100 \mathrm{mg}$ intraventricular (ANOVA, $p=0.029$; HolmSidak pairwise, $p<0.05$ ). There were no significant differences in blood pressure within groups over time or between groups at each time (ANOVA). Values are means $\pm \mathrm{SD}$

Assessment of the nimodipine-PLGA microparticles in humans with SAH is required and a dose-finding study is underway (NCT01893190). One question is what dose to use. An option would be to conduct detailed comparisons of the safety and efficacy of enteral nimodipine and nimodipine-PLGA microparticles in animal models and then select the lowest efficacious and safe dose. The limitations of this approach include that animal models that reproduce brain injury and DCI similar to what occurs after human aneurysmal SAH are not well characterized or even nonexistent. The current dog efficacy study assessed angiographic vasospasm, but this is an imperfect surrogate measure of DCI [7]. No dose response may be detected, as was the case in the human nimodipine studies, in which the highest safe dose was approved for use. Finally, we have already conducted safety studies that demonstrate a dose-dependent, clinically undetectable, and resolving inflammatory reaction to nimodipine-PLGA microparticles that is already a well-known effect of PLGA, a chemical already implanted intracranially as a dural substitute. Therefore, we adopted an approach is to conduct a dosefinding, safety, and pharmacokinetic study in humans starting with a dose well below the NOAEL, as recommended by regulatory agencies. It also may be warranted to use the current approach to study other drugs to treat other conditions that are self-limited and confined to preformed body cavities. 
Acknowledgments Daniel Hänggi and Nima Etminan are scientific advisors for Edge Therapeutics, Inc.; Mark Johnson and M. Melissa Peet are employees of MPI Research. Tom Tice, Kevin Burton, Bruce Hudson, Michele Turner, Parissa Heshmati, and Cara Davis are employees of Evonik Degussa Corp. Angela Stella is an employee of Pharmaceutical Associates, LLC. R. Loch Macdonald is Chief Scientific Officer of Edge Therapeutics, Inc. and received grant support from the Physicians Services Incorporated Foundation, Brain Aneurysm Foundation, Canadian Institutes for Health Research, and the Heart and Stroke Foundation of Canada. H. Faleck is an employee of Edge Therapeutics, Inc.

Required Author Forms Disclosure forms provided by the authors are available with the online version of this article.

\section{References}

1. van der Worp HB, Howells DW, Sena ES, et al. Can animal models of disease reliably inform human studies? PLoS Med. 2010;7(3): e1000245.

2. Begley DJ. Delivery of therapeutic agents to the central nervous system: the problems and the possibilities. Pharmacol Ther. 2004;104(1):29-45.

3. Gabathuler R. Approaches to transport therapeutic drugs across the blood-brain barrier to treat brain diseases. Neurobiol Dis. 2010;37(1):48-57.

4. Brem H, Piantadosi S, Burger PC, et al. Placebo-controlled trial of safety and efficacy of intraoperative controlled delivery by biodegradable polymers of chemotherapy for recurrent gliomas. The Polymer-brain Tumor Treatment Group. Lancet. 1995;345(8956): 1008-1012.

5. Al-Khindi T, Macdonald RL, Schweizer TA. Cognitive and functional outcome after aneurysmal subarachnoid hemorrhage. Stroke. 2010;41(8):e519-e536.

6. Nieuwkamp DJ, Setz LE, Algra A, Linn FH, de Rooij NK, Rinkel GJ. Changes in case fatality of aneurysmal subarachnoid haemorrhage over time, according to age, sex, and region: a meta-analysis. Lancet Neurol. 2009;8(7):635-42.

7. Macdonald RL. Delayed neurological deterioration after subarachnoid haemorrhage. Nat Rev Neurol. 2014;10(1):44-58.

8. Weyer GW, Nolan CP, Macdonald RL. Evidence-based cerebral vasospasm management. Neurosurg Focus. 2006;21(3):E8.

9. Dorhout Mees SM, Rinkel GJ, Feigin VL, et al. Calcium antagonists for aneurysmal subarachnoid haemorrhage. Cochrane Database Syst Rev. 2007(3):CD000277.

10. Towart R, Wehinger E, Meyer H, Kazda S. The effects of nimodipine, its optical isomers and metabolites on isolated vascular smooth muscle. Arzneimittelforschung. 1982;32(4):338-346.

11. Kasuya H, Onda H, Sasahara A, Takeshita M, Hori T. Application of nicardipine prolonged-release implants: analysis of 97 consecutive patients with acute subarachnoid hemorrhage. Neurosurgery. 2005;56(5):895-902

12. Barth $\mathrm{M}$, Capelle HH, Weidauer S, et al. Effect of nicardipine prolonged-release implants on cerebral vasospasm and clinical outcome after severe aneurysmal subarachnoid hemorrhage: a prospective, randomized, double-blind phase IIa study. Stroke. 2007;38(2): 330-336.
13. Bege N, Renette T, Endres T, Beck-Broichsitter M, Hanggi D, Kissel $\mathrm{T}$. In situ forming nimodipine depot system based on microparticles for the treatment of posthemorrhagic cerebral vasospasm. Eur J Pharm Biopharm 2013;84:99-105.

14. Toyota BD. The efficacy of an abbreviated course of nimodipine in patients with good-grade aneurysmal subarachnoid hemorrhage. J Neurosurg 1999;90:203-206.

15. Davson H, Welch K, Segal MB. Secretion of the cerebrospinal fluid. The Physiology and Pathophysiology of the Cerebrospinal Fluid. London: Churchill-Livingstone; 1987. p. 201.

16. Reagan-Shaw $\mathrm{S}$, Nihal M, Ahmad N. Dose translation from animal to human studies revisited. FASEB J. 2008;22(3):659-661.

17. Langley MS, Sorkin EM. Nimodipine. A review of its pharmacodynamic and pharmacokinetic properties, and therapeutic potential in cerebrovascular disease. Drugs. 1989;37(5):669-699.

18. Scriabine A, Battye R, Hoffmeister F, et al. Nimodipine. New Drugs Annual: Cardiovasc Drugs. 1985;3:197-218.

19. Zhou C, Yamaguchi M, Colohan AR, Zhang JH. Role of p53 and apoptosis in cerebral vasospasm after experimental subarachnoid hemorrhage. J Cereb Blood Flow Metab. 2005;25(5):572-582.

20. Yarnitsky D, Lorian A, Shalev A, et al. Reversal of cerebral vasospasm by sphenopalatine ganglion stimulation in a dog model of subarachnoid hemorrhage. Surg Neurol 2005;64(1):5-11.

21. Edwards D, Berry JJ. The efficiency of simulation-based multiple comparisons. Biometrics. 1987;43(4):913-928.

22. Menei P, Montero-Menei C, Venier MC, Benoit JP. Drug delivery into the brain using poly(lactide-co-glycolide) microspheres. Expert Opin Drug Deliv. 2005;2(2):363-376.

23. Vergouwen MD, Vermeulen M, de Haan RJ, Levi M, Roos YB. Dihydropyridine calcium antagonists increase fibrinolytic activity: a systematic review. J Cereb Blood Flow Metab. 2007;27(7):1293-1308.

24. Macdonald RL, Kassell NF, Mayer S, et al. Clazosentan to overcome neurological ischemia and infarction occurring after subarachnoid hemorrhage (CONSCIOUS-1): randomized, doubleblind, placebo-controlled phase 2 dose-finding trial. Stroke. 2008;39(11):3015-3021.

25. Etminan N, Vergouwen MD, Ilodigwe D, Macdonald RL. Effect of pharmaceutical treatment on vasospasm, delayed cerebral ischemia, and clinical outcome in patients with aneurysmal subarachnoid hemorrhage: a systematic review and meta-analysis. J Cereb Blood Flow Metab. 2011;31(6):1443-1451.

26. Pierot L, Aggour M, Moret J. Vasospasm after aneurysmal subarachnoid hemorrhage: recent advances in endovascular management. Curr Opin Crit Care 2010;16:110-116.

27. Dreier JP, Windmuller O, Petzold G, Lindauer U, Einhaupl KM, Dirnagl U. Ischemia triggered by red blood cell products in the subarachnoid space is inhibited by nimodipine administration or moderate volume expansion/hemodilution in rats. Neurosurgery. 2002;51(6): 1457-1465.

28. Sabri M, Ai J, Macdonald RL. Dissociation of vasospasm and secondary effects of experimental subarachnoid hemorrhage by clazosentan. Stroke. 2011;42(5):1454-1460.

29. Omeis I, Jayson NA, Murali R, Abrahams JM. Treatment of cerebral vasospasm with biocompatible controlled-release systems for intracranial drug delivery. Neurosurgery. 2008;63(6):1011-1019.

30. Zhang JH, Badaut J, Tang J, Obenaus A, Hartman R, Pearce WJ. The vascular neural network - a new paradigm in stroke pathophysiology. Nat Rev Neurol. 2012;8(12):711-716. 